Sharif University of Technology
Scientia Iranica
Transactions E: Industrial Engineering
hCIENTIA

Research Note

\title{
On the performance of median-based Tukey and Tukey-EWMA charts under rational subgrouping
}

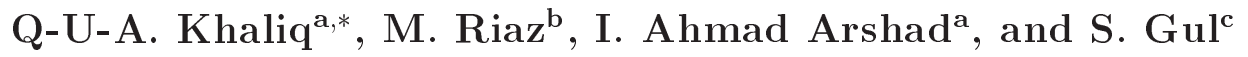 \\ a. Department of Statistics, Allama Iqbal Open University, Islamabad, Pakistan. \\ b. Department of Mathematics and Statistics, King Fahd University of Petroleum and Minerals, Dhahran 31261, Saudi Arabia. \\ c. Department of Education, Arid Agriculture University, Rawalpindi, Pakistan.
}

Received 29 October 2017; received in revised form 9 March 2019; accepted 29 April 2019

\section{KEYWORDS}

Average run length; Median;

Rational-subgroups;

Tukey chart;

Tukey EWMA chart.

\begin{abstract}
Control Chart (CC) is used to monitor specific problems and causes that arise during a process. These causes constantly change the process parameters until they are identified and removed. To this end, the techniques that present a true representation of the entire process should be used. Rational subgrouping is an essential concept in Statistical Process Control (SPC), which is usually overlooked by the practitioner. Hence, most of the manufacturing, engineering, and production processes give out output products in the form of batches over smaller time intervals. The aim of this study is to provide a median-based design for Tukey and Tukey-EWMA control charts under subgrouping. This study uses the idea of boxplot to monitor the process behavior. This study provided a brief discussion about selecting and forming subgroups from the process data. The performance of the median-based Tukey and Tukey-EWMA charts was evaluated using average, median, and standard deviation of run length as performance measures. This study considered subgroup sizes of $m=1.5$ and 10 at pre-specified ARL0 equal to 370. Real-life applications of the median-based Tukey designs were given to demonstrate their proper implementation in food manufacturing and hard-bake processes.
\end{abstract}

(C) 2021 Sharif University of Technology. All rights reserved.

\section{Introduction}

Tukey Control Chart (TCC) is a well-known individual control chart designed to monitor the skewed data using the concept of boxplot and is mainly based on the individual observations per period. Rational Subgrouping (R.S) is an essential concept in Statistical Process Control (SPC) and yet, it is frequently overlooked in some processes. In R.S, all goods and products are manufactured under a condition where only random effects are responsible for observed variations. It is

\footnotetext{
*. Corresponding author.

E-mail address: quratulainaineee@gmail.com (Q-U-A. Khaliq)
}

doi: $10.24200 /$ sci. 2019.5470 .1289 the process of organizing a similar group of products produced under similar circumstances. This process helps measure the variations between the subgroups rather than within subgroups, which are considered background noises. Subgroup size should be large enough to represent the overall variations when the process is in control. It is concerned with the collection and organization of numerical data. There are different pieces of literature available on Tukey designs including many of their modifications. Here, a brief review of some useful literature on the topic is provided.

\subsection{Literature review}

TCC is mainly an individual observation based control chart proposed by Alemi [1]. Borckardt et al. [2,3] applied the chart to serially dependent data. Torng and Lee [4] calculated the Average Run Length (ARL) 
values of the Tukey chart under several distributions. For small samples, TCC performed very well. Torng et al. [5] introduced the economic design of TCC. Lee [6] applied Asymmetrical Control Limits (ACLs) to TCC. In skewed distribution, ACL-TCC offered lower ARL values than SCL-TCC (symmetrical control limits). Sukparungsee [7] evaluated the performance of TCC; TCC performance was found superior to classical EWMA and Shewhart control charts. Lee et al. [8] applied the ACL to the economic design of TCC to get the optimum performance. Sukparungsee [9] applied ACL to Tukey chart to evaluate its performance under non-normal distributed data and Tukey chart outperformed $\mathrm{ARL}_{1}$.

Khaliq et al. [10] comparatively evaluated the performance of Tukey chart versus X/MR chart under several probability models and Tukey chart was the best choice in many cases. The study revealed that this chart was a good alternative to Shewhart and $\mathrm{X} / \mathrm{MR}$ chart for monitoring when data was skewed. Tercero-Gomez et al. [11] designed the Modified Tukey control Chart (MTCC) with smaller $\mathrm{ARL}_{1}$ values than TCC. Mekparyup et al. [12] introduced the adjusted design for Tukey chart. Mekparyup et al. [13] combined the features of the adjusted Tukey chart with those of an ARIMA (Auto-Regressive Integrated Moving Average) model to monitor the Dengue Hemorrhagic Fever (DHF).

Saithanu et al. [14] applied the sensitizing run rules scheme to Tukey chart. The results indicated that the run length performance of this scheme improved over the Tukey chart. Khaliq et al. [15] introduced the EWMA design for Tukey chart. The Tukey-EWMA design was more sensitive to small continuous shifts in process location than Tukey chart. This design is a good alternative to classical EWMA when the data follow a skewed distribution. Khaliq and Riaz [16] designed the CUSUM structure of Tukey chart for small and sustainable shifts. This design is the best alternative to classical CUSUM chart when the data follow the skewed distribution. For symmetric distribution, this design has a similar run length performance to classical CUSUM. Riaz et al. [17] introduced the mixed Tukey EWMA CUSUM design which was more sensitive to small and moderate cases of shifts.

All of the above-mentioned charts were designed to deal with the individual observations. The current study designed median-based TCC and TukeyEWMA Control Chart (EWMA-TCC) for subgroupbased observations. These median-based TCC and EWMA-TCC charts enjoy a variety of applications in different areas such as business, technology, management, education, manufacturing, accounting, finance, engineering, and service sectors. We will discuss two applications in detail in Section 5 with emphasis on food manufacturing and hard-bake processes.
The rest of the paper is organized as follows: Section 2 discusses the significance of the subgrouping in control chart. Section 3 presents median-based Tukey and EWMA-TCC charts under R.S. Section 4 provides performance analysis of the charts. Section 5 includes two real applications. Section 6 summarizes and concludes the study.

\section{Significance of subgrouping in control charts}

Rational Subgrouping (R.S) is the fundamental spirit of any application of process behavior chart. It does not follow the procedure to form the subgroups. It will be nothing more than wall-paper. As an alternative, the observations compromising the subgroup must follow the process at a short-time interval and show how it fluctuates over time. The size time interval established an individual process basis to reduce the chance of a special cause happening in the subgroup. Control charts are generally based on more than one sample observation, $m>1$, selected at a fixed length of sampling interval, say $l$, and the rational-subgroup idea of sampling implies that sampling must be done subsequently and any change to the process will occur among samples and affect the entire sample. However, if the length of a transient shift $t$ is smaller than that of $l$, then it seems that it may be useful to disperse the samples over the interval $l$. Here, the chance for the transient shift to occur increases. Sampling and subgrouping should be carried out with care. According to Nelson [18], "the rational subgroup is basically a sample in which random effects are responsible for the observed variation during the product production".

Sefik [19] completely discussed the importance of subgrouping in the process control chart. Nelson [20] discussed the properties of R.S in the following. The observations among a subgroup constitute a single and stable process. If the subgroups following the multiple process stream with a particular cause happen continually within the subgroup, there will be more variations within sample rather than between subgroup averages and these variations may lead to widening the control limits and lack of sensitivity to the process shifts. "Western Electric Role Test-vii fifteensuccessive points within one sigma of center line are helpful in detecting this condition". The subgroups are formed non-randomly from the observations in a timeordered sequence. As an alternative, the observations comprising the subgroup must display the process at a short time interval and show how it fluctuates over time. The size time interval demonstrated an individual process basis to reduce the chance of a special cause happening in the subgroup. Hillier [21,22] designed adjusted limits of Shewhart-type chart in retrospective and future testing stages. These limits were used to ensure the future desired subgroup size with pre- 
specified type-I error. According to Wheeler and David [23], subgroups should be logically homogenous by minimizing the variation within subgroup by trying to maximize the variations between subgroups. It should be noted that before forming the subgroup, the samples which comprise the subgroup must be homogenous and independent over time. They must be collected in a time-order sequence in a stable process. Quenvedo et al. [24] applied an iterative procedure to classical $X$ bar and $R$ chart to set the control limits of the respective chart better. Djauhari et al. [25] evaluated the performance of the multivariate chart when the subgroup size was too small. Abbasi et al. [26] modified the classical EWMA design to improve the sensitivity of a memory chart. Mahmood et al. [27] introduced the joint memory structure of the control chart to monitor location and spread. Ajadi and Riaz [28] designed memory-type control charts to monitor the subgroup data. Abujiya et al. [29] developed the variance chart to monitor dispersion in the process monitoring. Mukherjee [30] applied the joint monitoring scheme of location and spread to the non-parametric EWMA chart based on the subgroup samples. Ansorena [31] used SPC control design to monitor the quality of seaport services. Hussain et al. [32] designed the interquartile range-based EWMA chart to monitor the continuous tank rector process. Abtew et al. [33] applied the SPC chart in the sewing section of garment industry. Rational subgroups of the samples were first formed to evaluate the process performance. Huberts et al. [34] introduced a method for continuously updating the control limits of control chart when data were provided in the subgroup form.

\subsection{Two common methods for constructing rational subgroups}

i. The method for R.S provides a pictorial display of the system at each point in time wherein an observation is collected. It is used when the main objective of the control chart is to identify the process shift. It diminishes the chance of inconsistency due to special causes within a sample and it increases the chance of variability among the samples if the assignable cause is present. It additionally offers a higher estimate of the standard deviation of the manner in the case of variable control charts;

ii. Each sample comprises units of products that illustrate all the units that have been produced since the last sample was taken. Basically, every subgroup is an unsystematic sample of the entire process output over the sampling interval. This technique of R.S is frequently used when the control chart is employed to draw conclusions about the acceptance of all units of products that have been produced since the last sample. Indeed, if the process shifts to an out-of-control state and then, back in control again between samples, it is sometimes argued that the picture technique of R.S will be unsuccessful against these types of changes; therefore, the technique sample scheme must be used (cf., Montgomery [35]).

\section{Median-based Tukey and EWMA-TCC under rational subgroup}

Let $X_{i_{j}}$ be independent observations collected over time from a normal process, $i=1,2,3, \cdots, n$ and $j=1,2,3, \cdots, m$. That is, we have $m$ subgroups, each of which is characterized by size $n$. Now, three quartiles $\left(q_{1}, q_{2}, q_{3}\right)$ and interquartile range $(i q r)$ for all the $m$ subgroups are computed which are presented in the following:

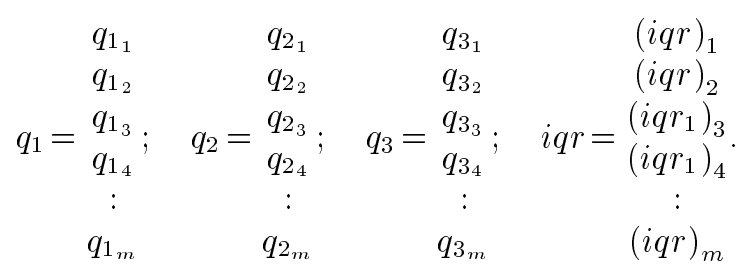

Let $\tilde{q}_{1}, \tilde{q}_{2}, \tilde{q}_{3}$, and $i \tilde{q} r$ be the medians of first, second, third, and interquartile range of the subgroup data sets, respectively.

Then, the control limits of TCC are given as follows:

$$
\begin{aligned}
& L C L=\tilde{q}_{1}-L(i \tilde{q} r), \\
& C L=\tilde{q}_{2}, \\
& U C L=\tilde{q}_{3}+L(i \tilde{q} r),
\end{aligned}
$$

where $L$ is the control limits coefficient and it is set according to pre-specified $\mathrm{ARL}_{0}$. The median $\left(q_{2}=\right.$ $\left.\tilde{x}_{j},=1,2,3, \cdots\right)$ ) of these values will be used as the plotting statistic for the TCC chart.

The plotting statistic for EWMA-TCC is as follows:

$$
G_{j}=\lambda \tilde{x}_{j}+(1-\lambda) G_{j-1} .
$$

The variance of EWMA-TCC statistic is given below:

$$
\operatorname{Var}\left(G_{j}\right)=\frac{i \tilde{q} r\left(\lambda\left(1-(1-\lambda)^{2 j}\right)\right)}{2-\lambda},
$$

where $\lambda$ is the weighting parameter and it lies between 0 and 1 . For $\lambda=1$, it exhibits the most recent observation and becomes the special case of TCC. The initial value of $G_{i}$ (i.e., $G_{0}$ ) is set equal to the overall median. 
The time varying control limits of EWMA-TCC are given as follows:

$$
\begin{aligned}
& L C L=\tilde{q}_{1}-L_{t}(i \tilde{q} r) \sqrt{\frac{\lambda\left(1-(1-\lambda)^{2 j}\right)}{2-\lambda},} \\
& C L=\tilde{q}_{2}, \\
& U C L=\tilde{q}_{3}+L_{t}(i \tilde{q} r) \sqrt{\frac{\lambda\left(1-(1-\lambda)^{2 j}\right)}{2-\lambda}},
\end{aligned}
$$

where $L_{t}$ is the control limits coefficient taking into account the time varying feature. These control limits are known as time varying control limits which are dependent upon the $j$. When $j \rightarrow \infty,\left(1-(1-\lambda)^{2 j}\right) \rightarrow$ 1 and the time varying limits turn into the asymptotic limits given as follows:

$$
\begin{aligned}
& L C L=\tilde{q}_{1}-L_{t}(i \tilde{q} r) \sqrt{\frac{\lambda}{2-\lambda}}, \\
& C L=\tilde{q}_{2}, \\
& U C L=\tilde{q}_{3}+L_{t}(i \tilde{q} r) \sqrt{\frac{\lambda}{2-\lambda}} .
\end{aligned}
$$

\section{Performance evaluations and analysis}

A sequence of points plotted on a chart until an outof-control signal is identified is known as a run and a series of points in a run are named as Run Lengths (RLs). Typically, control RL is expected to be higher, while out-of-control RL is anticipated to be as small as possible. One may see Chakraborti [36] for more useful discussion on RL.

Several measures based on RL are presented in literature to assess the performance of the chart, some are used for specific shifts and others for the overall shifts in a process. For this study, only specific shift measures including average, standard deviation and median RL are considered. The details of these measures are provided in the following:
Average Run Length (ARL): It is generally used to assess the performance of a chart for a specific shift value. It represents the average number of points plotted on a chart until an out-of-control signal is identified. There are two famous terms used in the control chart for the aforesaid purpose, termed as in control and out of control ARLs. The in and out of control measures are denoted by $\mathrm{ARL}_{0}$ and $\mathrm{ARL}_{1}$. A chart showing smaller $\mathrm{ARL}_{1}$ on a particular shift value is considered to be more efficient than other competing charts. An estimate of ARL may be given as follows:

$$
A R L=\sum_{j}^{k}(R L)_{j} / k .
$$

Standard Deviation Run Length (SDRL): The dispersion of RL may be observed by Variance and Standard Deviation of RL. This shows how much average variation is present in the particular control chart RL values. An estimate of SDRL may be given as follows:

$$
S D R L=\sqrt{\sum_{j}^{k}(R L)_{j}^{2} / k-\left\{\left(\sum_{j}^{k}(R L)_{j} / k\right)^{2}\right\}} .
$$

Median Run Length (MRL): The distribution of RL is mostly skewed and hence, median of RL is another the most suitable choice. Median, being a wonderful and robust measure for outliers, is a more detailed performance indicator of a chart and it is defined as follows:

$$
M R L=\text { Median (RL). }
$$

Using Monte Carlo simulations, we have computed the aforementioned RL properties of both medianbased TCC and EWMA-TCC charts. The results are reported in Tables 1, 2, 3 in the form of ARL, MRL, and SDRL using several subgroup values at $\mathrm{ARL}_{0}$ $=370$. Figure 1 presents RL curves of Tukey and Tukey-EWMA at several subgroup sizes $m$ with fixed

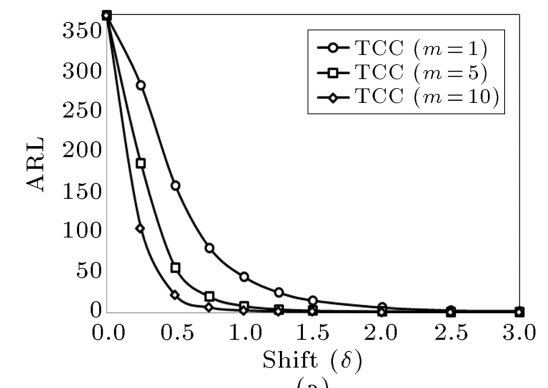

(a)

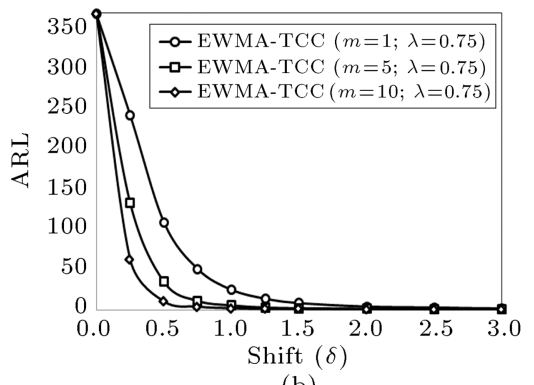

(b)

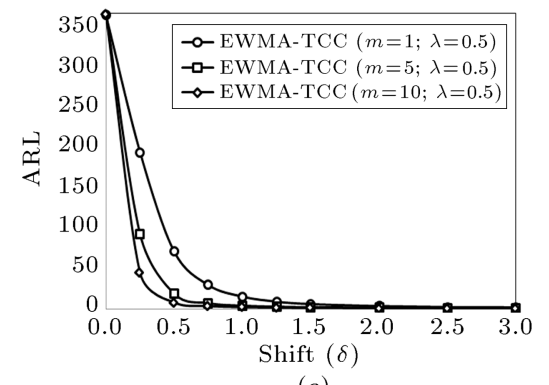

(c)

Figure 1. Average Run Length (ARL) performance of Tukey Control Chart (TCC) and EWMA-TCC at different subgroup sizes $(m=1,5,10)$ for (a) TCC, (b) EWMA-TCC at $\lambda=0.7$, and (c) EWMA-TCC at $\lambda=0.5$. 
Table 1. Average Run Length (ARL) performance of Tukey Control Chart (TCC) and EWMA-TCC using different subgroup sizes $(m)$.

\begin{tabular}{|c|c|c|c|c|c|c|c|c|c|}
\hline \multirow[b]{2}{*}{$\delta / m$} & \multicolumn{3}{|c|}{ TCC } & \multicolumn{3}{|c|}{ EWMA-TCC $(\lambda=0.75)$} & \multicolumn{3}{|c|}{ EWMA-TCC $(\lambda=0.5)$} \\
\hline & $m=1$ & $m=5$ & $m=10$ & $\boldsymbol{m}=\mathbf{1}$ & $m=5$ & $m=10$ & $m=1$ & $\boldsymbol{m}=\mathbf{5}$ & $m=10$ \\
\hline 0 & 370.12 & 370.1404 & 370.52 & 370.00 & 370.14 & 370 & 369.124 & 371.3 & 370.41 \\
\hline 0.25 & 283.13 & 178.69 & 102.38 & 235.29 & 125.41 & 63.01 & 196.62 & 83.83 & 40.17 \\
\hline 0.5 & 158.21 & 56.10 & 21.72 & 109.47 & 35.06 & 10.68 & 71.959 & 19.02 & 8.08 \\
\hline 0.75 & 80.33 & 19.57 & 6.38 & 51.01 & 11.18 & 4.042 & 29.88 & 7.0938 & 3.53 \\
\hline 1 & 44.27 & 8.15 & 2.69 & 25.04 & 5.57 & 2.14 & 15.34 & 3.87 & 2.17 \\
\hline 1.25 & 25.10 & 4.06 & 1.59 & 13.94 & 2.90 & 1.46 & 8.97 & 2.65 & 1.621 \\
\hline 1.5 & 15.03 & 2.39 & 1.18 & 8.55 & 2.04 & 1.18 & 5.92 & 2.0135 & 1.29 \\
\hline 2 & 6.28 & 1.29 & 1.009 & 4.11 & 1.31 & 1.01 & 3.42 & 1.405 & 1.0307 \\
\hline 2.5 & 2.55 & 1.05 & 1 & 2.99 & 1.05 & 1 & 2.11 & 1.16 & 1.00 \\
\hline 3 & 1.97 & 1 & 1 & 1.79 & 1 & 1 & 1.861 & 1.01 & 1 \\
\hline 4 & 1.19 & 1 & 1 & 1.18 & 1 & 1 & 1.29 & 1 & 1 \\
\hline
\end{tabular}

Table 2. Median Run Length (MRL) performance of Tukey Control Chart (TCC) and EWMA-TCC using different subgroup sizes $(m)$.

\begin{tabular}{|c|c|c|c|c|c|c|c|c|c|}
\hline \multirow[b]{2}{*}{$\delta / m$} & \multicolumn{3}{|c|}{ TCC } & \multicolumn{3}{|c|}{ EWMA-TCC $(\lambda=0.75)$} & \multicolumn{3}{|c|}{ EWMA-TCC $(\lambda=0.5)$} \\
\hline & $m=1$ & $m=5$ & $m=10$ & $m=1$ & $m=5$ & $m=10$ & $m=1$ & $m=5$ & $m=10$ \\
\hline 0 & 256 & 195 & 156 & 257 & 201 & 166 & 256 & 188 & 155 \\
\hline 0.25 & 191 & 99 & 65 & 164 & 89.5 & 41 & 133 & 51 & 28 \\
\hline 0.5 & 108 & 31 & 14 & 76 & 24 & 7 & 50 & 12 & 6 \\
\hline 0.75 & 57 & 12 & 4 & 36 & 8 & 3 & 21 & 5 & 3 \\
\hline 1 & 30 & 5 & 2 & 18 & 4 & 2 & 11 & 3 & 2 \\
\hline 1.25 & 17 & 3 & 1 & 10 & 3 & 1 & 7 & 2 & 2 \\
\hline 1.5 & 11 & 2 & 1 & 6 & 2 & 1 & 5 & 2 & 1 \\
\hline 2 & 4 & 1 & 1 & 3 & 1 & 1 & 3 & 1 & 1 \\
\hline 2.5 & 2 & 1 & 1 & 3 & 1 & 1 & 2 & 1 & 1 \\
\hline 3 & 1 & 1 & 1 & 2 & 1 & 1 & 2 & 1 & 1 \\
\hline 4 & 1 & 1 & 1 & 1 & 1 & 1 & 1 & 1 & 1 \\
\hline
\end{tabular}

Table 3. Standard Deviation Run Length (SDRL) performance of Tukey Control Chart (TCC) and EWMA-TCC using different subgroup sizes $(m)$.

\begin{tabular}{|c|c|c|c|c|c|c|c|c|c|}
\hline \multirow[b]{2}{*}{$\delta / m$} & \multicolumn{3}{|c|}{ TCC } & \multicolumn{3}{|c|}{ EWMA-TCC $(\lambda=0.75)$} & \multicolumn{3}{|c|}{ EWMA-TCC $(\lambda=0.5)$} \\
\hline & $m=1$ & $m=5$ & $m=10$ & $m=1$ & $m=5$ & $m=10$ & $m=1$ & $m=5$ & $m=10$ \\
\hline 0 & 376.64 & 374.37 & 370.173 & 367.74 & 528.70 & 369.21 & 373.40 & 564.80 & 469.25 \\
\hline 0.25 & 279.72 & 282.92 & 129.18 & 246.97 & 138.16 & 76.82 & 194.63 & 131.42 & 53.33 \\
\hline 0.5 & 157.79 & 77.96 & 23.47 & 110.36 & 34.32 & 10.99 & 70.062 & 20.30 & 6.88 \\
\hline 0.75 & 81.67 & 25.82 & 6.24 & 50.78 & 9.59 & 3.079 & 28.52 & 5.86 & 2.049 \\
\hline 1 & 42.73 & 9.17 & 2.23 & 23.84 & 4.17 & 1.23 & 13.41 & 2.40 & 0.97 \\
\hline 1.25 & 25.21 & 4.07 & 0.99 & 12.86 & 1.78 & 0.69 & 6.89 & 1.31 & 0.64 \\
\hline 1.5 & 14.65 & 2.00 & 0.46 & 7.52 & 1.187 & 0.39 & 4.233 & 0.87 & 0.47 \\
\hline 2 & 5.84 & 0.62 & 0.09 & 3.038 & 0.53 & 0.099 & 1.87 & 0.53 & 0.17 \\
\hline 2.5 & 4.52 & 0.24 & 0.08 & 1.56 & 0.235 & 0 & 0.98 & 0.33 & 0.02 \\
\hline 3 & 1.43 & 0.08 & 0.05 & 0.95 & 0.099 & 0 & 0.74 & 0.13 & 0.01 \\
\hline 4 & 0.47 & 0 & 0.014 & 0.42 & 0 & 0 & 0.47 & 0 & 0 \\
\hline
\end{tabular}




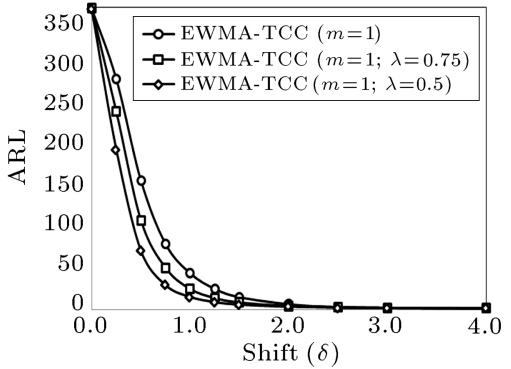

(a)

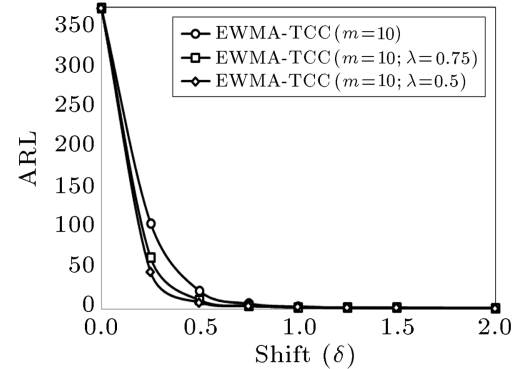

(b)

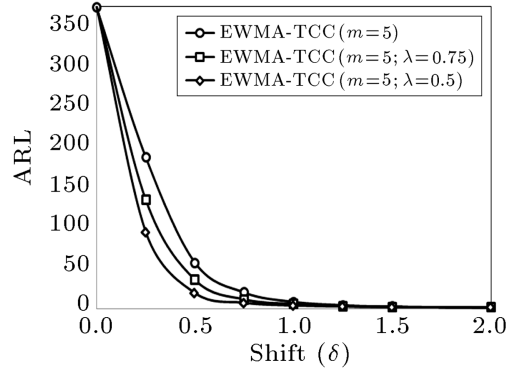

(c)

Figure 2. Comparative performance of EWMA-TCC and Tukey Control Chart (TCC) at several $\lambda$ and subgroup sizes for: (a) TCC and EWMA-TCC $(\lambda=0.75$ and 0.5$)$ designs at $m=1$; (b) TCC and EWMA-TCC $(\lambda=0.75$ and 0.5$)$ designs at $m=5$, and (c) TCC and EWMA-TCC $(\lambda=0.75$ and 0.5$)$ designs at $m=10$.

$\lambda$. Moreover, Figure 2 presents ARL curves of TCC and EWMA-TCC charts at several $\lambda$ and $m$. The performance analysis of these control charts supports the following findings (cf., Tables $1-3$ and Figures 1 and 2):

\section{TCC analysis}

- With an increase in the size of subgroups, the $\mathrm{ARL}_{1}$ of median-based TCC exhibits a decreasing pattern. For example, TCCs with $\mathrm{ARL}_{1}$ values are 178.13 and 102.38 for $m=5$ and 10 at $\delta=.25$. A similar pattern is shown in Figure 1(a);

- The larger the subgroup size, the smaller the MRL and SDRL values for median-based TCC. For instance, MRL values of the proposed chart are 191, 99, 65, while SDRL values are 282.91, 279.72, 129.18 at $\delta=0.25$ and $m=1,5$, and 10 (cf., Tables 2 and 3 ). Based on these results, when the subgroup size varies from 1 to 10 , MRL and SDRL values keep getting smaller.

\section{EWMA-TCC analysis}

- The subgroup size and $\lambda$ affect the performance of the median-based EWMA-TCC. As the subgroup size $m$ increases, the median-based EWMA-TCC chart exhibits more sensitivity towards shifts. For example, $\mathrm{ARL}_{1}$ values of EWMA-TCC are 243.29, 125.41 , and 63.016 at $\delta=0.25, \lambda=0.75 \mathrm{using}$ subgroup size 1,5 , and 10 . It is clear that the subgroup size increases the sensitivity of EWMATCC for small to moderate shifts (cf., Table 1). Moreover, in Figure 1(a) and (b), EWMA-TCC design offers a steeper $\mathrm{ARL}_{1}$ curve at $\lambda=0.5$ and $m=10$;

- The large subgroup size ensures smaller ARL, MRL, and SDRL values of median-based EWMA-TCC. For example, MRL values of EWMA-TC are 164, 89.5, and 41, while the SDRL values are 246.97, 138.16 , and 73.82 at $\delta=0.25, \lambda=0.75$, and $m=1$, 5 , and 10. A similar outcome may be observed for the other values of $\lambda$ (cf., Tables 2 and 3 ).
- The median-based EWMA-TCC design (at $\lambda=0.75$ and 0.5 ) shows smaller $\mathrm{ARL}_{1}, \mathrm{MRL}$, and SDRL values than the median-based TCC for small to moderate shifts and they are seen in Tables 1-3 and Figure 2.

\section{The real applications}

This section considers two real life datasets to illustrate subgroup-based TCCs. The details and application of both datasets are given below:

Application 1: Food manufacturing process. The food industry has started showing tendency towards the utilization of nanotechnology. The nanotechnology plays its role in food ingredients, food packaging, water purification; improving mechanical strength; reducing weight; increasing heat resistance; improving barrier against oxygen, carbon dioxide, ultra-violet radiation, moisture, and volatiles of food packaging materials. Packaging is the process of enclosing the meals material in a container to ensure the delivery of products in fine circumstances to the customer for final use. Therefore, proper packaging plays an essential role in enhancing the image of company product. Packaging protects the meals and permits it to reach the customer in a hygienic and safe condition. The use of protecting coatings and appropriate packaging by the food industry can surely increase the shelf life of food product (cf. [37]). Ahmed et al. [38] and Razzaq et al. [39] discussed the importance of nano-technology in several manufacturing industries.

Figure 3 displays manufacturing setting of different nanotechnology materials that are produced by five different machines. The different subgroup arrangements are found after packaging food products. We have considered formation of the subgroups during food packaging process and collected data from an ongoing process in a firm (cf., Figure 3 ) located in RWP Pakistan. A group of 50 samples was selected from the process, each characterized by batch size ten. For this 


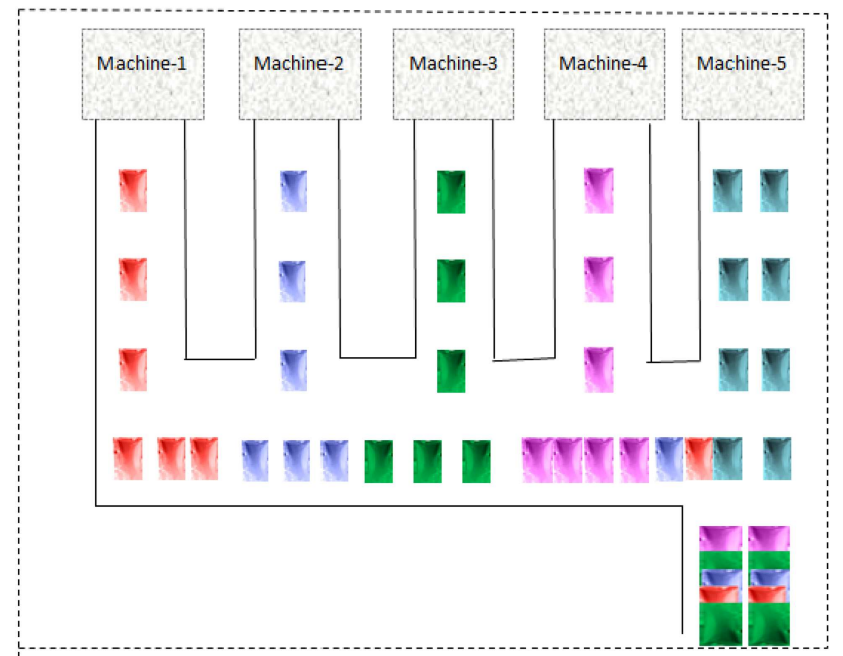

Figure 3. Procedural flow of forming subgroup during the packaging process.

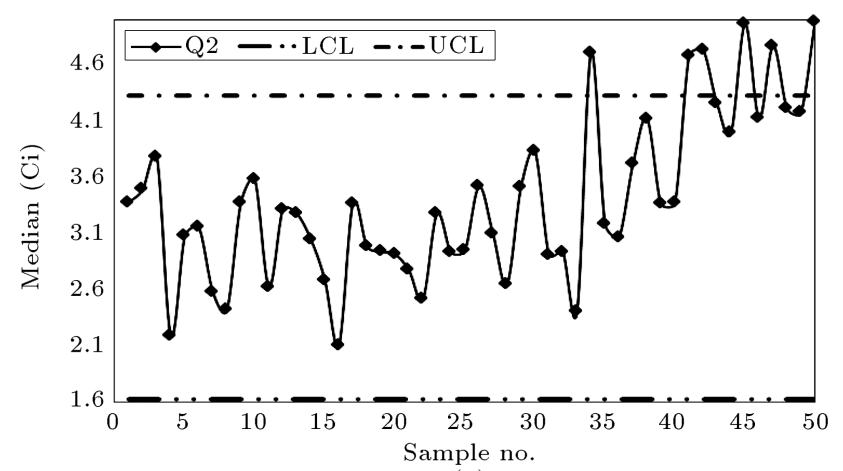

(a)

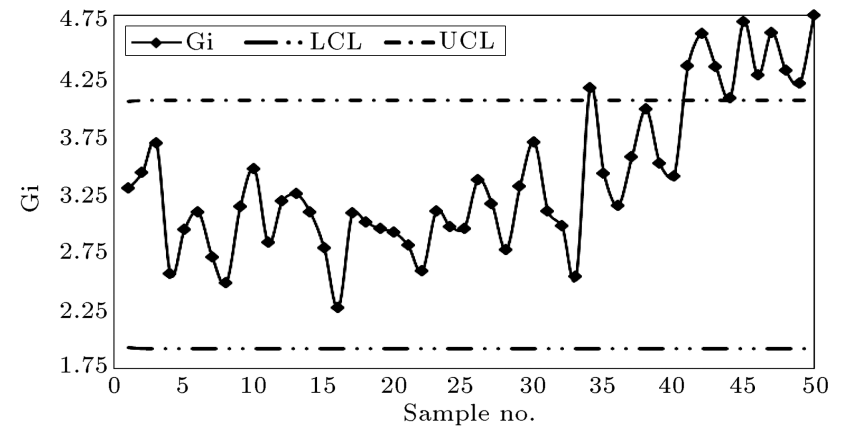

(b)

Figure 4. Implementation of charts on manufacturing data: (a) TCC and (b) EWMA-TCC.

dataset, we have constructed two control charts (TCC and EWMA-TCC (at $\lambda=.25)$ ) as shown in Figure 4.

Clearly, TCC and EWMA-TCC offer six and ten out-of-control signals, respectively. It shows that the process has a mix up of both small and large shifts and our two charts are helpful in alarming these changes in the process.

Application 2: Hard-bake process. A hard-bake process was used in conjunction with the photolithog-

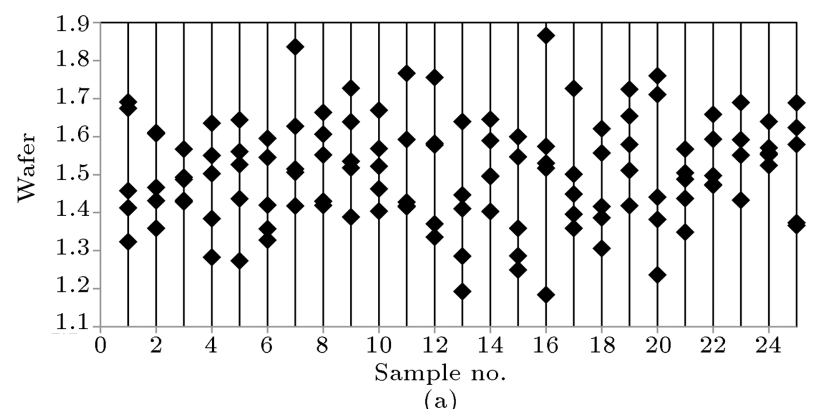

(a)
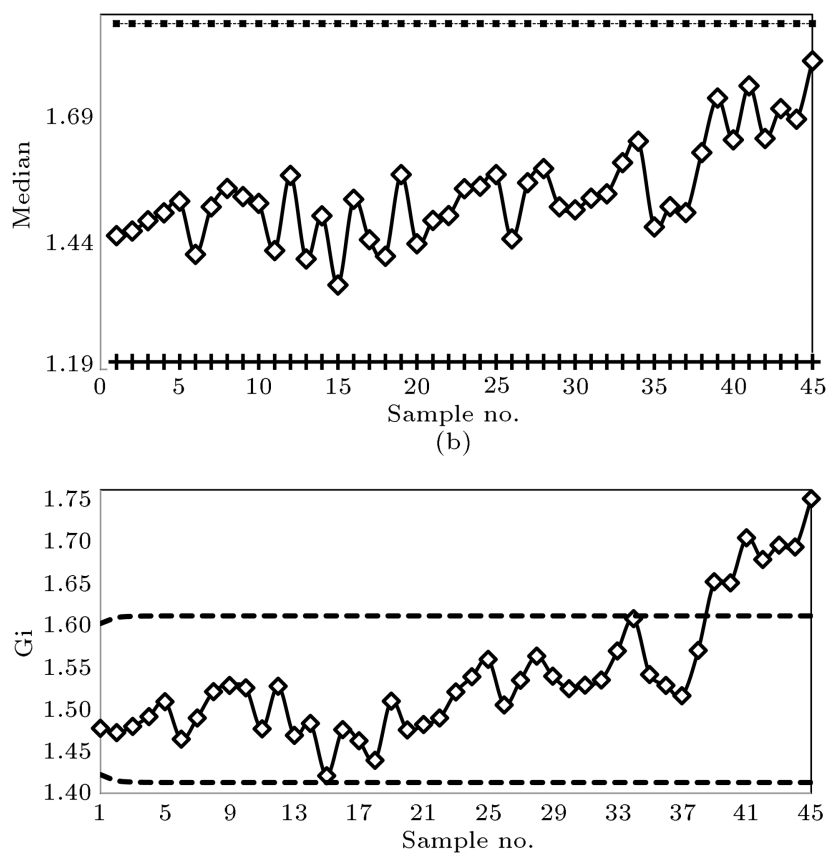

(c)

Figure 5. Implementation of charts on wafer data for (a) display of data, (b) TCC, and (c) EWMA-TCC.

raphy in semiconductor manufacturing. Our objective is to establish statistical control of the flow width that resists this process. The data on the mentioned variable was taken from Montgomery [35] and we intend to construct TCC and EWMA-TCC. Twentyfive samples, each with five wafers, were taken when the process was of in-control type. The time interval of subgroups is one hour. The display of the wafer data is given in Figure 5. We have implemented both TCC and EWMA-TCC $(\lambda=.25)$ charts for the mentioned dataset. TCC offered no points in the out-of-control state, while EWMA-TCC detected eight points in same state. This finding supports the occurrence of smaller shifts that are not captured by TCC, but EWMA-TCC successfully signaled them.

\section{Summary and conclusions}

This study investigated the performance of medianbased Tukey and Tukey-EWMA charts using Rational Subgrouping (R.S) concept. The manufacturing and 
industrial data mostly formed in the form of batches at equal time intervals. Traditional Tukey-type charts were designed to monitor the individual observations over time. In the current study, the design of the median-based Tukey and Tukey-EWMA (TCC and EWMA-TCC) charts was presented using subgroups. The Average Run Length (ARL), Standard Deviation Run Length (SDRL), and Median Run Length (MRL) measures were employed to evaluate the performance ability. These median-based Tukey Control Chart (TCC) and EWMA-TCC charts exhibited very effective performance under R.S. Two real life cases were also presented to show the practical application in real processes such as manufacturing, production, and packaging under the subgroups data. The scope of the idea may be extended easily to the Tukey-CUSUM design following the same line of action.

\section{Acknowledgments}

The authors are thankful to the anonymous reviewer for the constructive comments that helped improve the last version of the paper. The author, Muhammad Riaz, would also like to acknowledge the research facilities provided by the Deanship of Scientific Research (DSR) at King Fahd University of Petroleum $\&$ Minerals (KFUPM).

\section{Nomenclature}

$\begin{array}{ll}\text { ACL } & \text { Asymmetrical Control Limits } \\ \text { ARL } & \text { Average Run Length } \\ \text { CUSUM } & \text { Cumulative Sum } \\ \text { CL } & \text { Control Limits }\end{array}$

EWMA-TCC Exponentially Weighted Moving Average Tukey Control Chart

Iqr Interquartile range

LCL Lower Control Limit

MEC-TCC Mixed Tukey EWMA-CUSUM

S.G Sub-Grouping

ARIMA Auto-Regressive Integrated Moving Averages

SDRL Standard Deviation Run Length

SPC Statistical Process Control

MDRL Median RL

TCC Tukey Control Chart

MTCC $\quad$ Modified TCC

RL Run Length

DHF Dengue Hemorrhagic Fever

SCL Symmetrical Control Limits

$\mathrm{ARL}_{0} \quad$ In control ARL

$\mathrm{ARL}_{1} \quad$ Out of control ARL $\tilde{q}_{1}$

$\tilde{q}_{2}$

$i \tilde{q} r$

$X$

$\tilde{q}_{3}$

$L$

R.S

$L_{t}$

Median of first quartile

Median of second quartile

Median interquartile range

Quality characteristics

Median third quartile

Control limits coefficient

Rational Subgrouping

EWMA's control limits coefficient

\section{References}

1. Alemi, F. "Tukey's control chart", Quality Management in Healthcare, 13(4), pp. 216-221 (2004).

2. Borckardt, J.J., Nash, M.R., Hardesty, S., Herbert, J., Cooney, H., and Pelic, C. "An empirical evaluation of Tukey's control chart for use in health care and quality management applications", Quality Management in Healthcare, 14(2), pp. 112-115 (2005).

3. Borckardt, J.J., Pelic, C., Herbert, J., Borckardt, D., Nash, M.R., Cooney, H., and Hardesty, S. "An autocorrelation-corrected nonparametric control chart technique for health care quality applications", Quality Management in Healthcare, 15(3), pp. 157-162 (2006).

4. Torng, C.C. and Lee, P.H. "ARL performance of the Tukey's control chart", Communications in StatisticsSimulation and Computation, 37(9), pp. 1904-1913 (2012).

5. Torng, C.C., Lee, P.H., and Tseng, C.C. "An economic design of Tukey's control chart", Journal of the Chinese Institute of Industrial Engineers, 26(1), pp. 53-59 (2009).

6. Lee, P.H. "The effects of Tukey's control chart with asymmetrical control limits on monitoring of production processes", African Journal of Business Management, 5(11), pp. 4044-4050 (2011).

7. Sukparungsee, S. "Robustness of Tukey's control chart in detecting a changes of parameter of skew distributions", International Journal of Applied Physics and Mathematics, 2(5), p. 379 (2012).

8. Lee, P.H., Kuo, T.I., and Lin, C.S. "Economically optimum design of Tukey's control chart with asymmetrical control limits for controlling process mean of skew population distribution", European Journal of Industrial Engineering, 7(6), pp. 687-703 (2013).

9. Sukparungsee, S. "Asymmetric Tukey's control chart robust to skew and non-skew process observation", International Journal of Mathematical, Computational Science and Engineering, 7(8), pp. 51-54 (2013).

10. Khaliq, Q.U.A., Riaz, M., and Alemi, F. "Performance of Tukey's and individual/moving range control 
charts", Quality and Reliability Engineering International, 31(6), pp. 1063-1077 (2014).

11. Tercero-Gomez, V., Ramirez-Galindo, J., CorderoFranco, A., Smith, M., and Beruvides, M. "Modified Tukey's control chart", Communications in StatisticsSimulation and Computation, 41(9), pp. 1566-1579 (2012).

12. Mekparyup, J., Kornpetpanee, S., and Saithanu, K. "The performance of the adjusted Tukey's control chart under asymmetric distributions", Global Journal of Pure and Applied Mathematics, 10(5), pp. 719-724 (2014).

13. Mekparyup, J., Kornpetpanee, S., and Saithanu, K. "The adjusted Tukey's control chart with MADM", International Journal of Applied Environmental Sciences, 9(4), pp. 2063-2075 (2014).

14. Saithanu, K., Nojit, T., and Mekparyup, J. "Integration of sensitizing runs rules and Tukey's control chart in monitoring gamma distribution", Global Journal of Pure and Applied Mathematics, 11(3), pp. 269-272 (2015).

15. Khaliq, Q.U.A., Riaz, and Ahmad, S. "On designing a new Tukey-EWMA control chart for process monitoring", The International Journal of Advanced Manufacturing Technology, 82(1-4), pp. 1-23 (2016).

16. Khaliq, Q.U.A. and Riaz, M. "Robust Tukey-CUSUM control chart for process monitoring", Quality and Reliability Engineering International, 32(3), pp. 933948 (2016).

17. Riaz, M., Khaliq, Q.U.A., and Gul, S. "Mixed Tukey EWMA-CUSUM control chart and its applications", Quality Technology \& Quantitative Management, 14(4), pp. 378-411 (2017).

18. Nelson, L.S. "Setting up a control chart using subgroups of varying sizes", Journal of Quality Technology, 22(3), pp. 245-246 (1990).

19. Sefik, M. "Importance of the rational subgroups in designing control charts", Computers \& Industrial Engineering, 35(1-2), pp. 205-208 (1998).

20. Nelson, L.S. "Control charts-rational subgroups and effective applications", Journal of Quality Technology, 20(1), pp. 73-75 (1988).

21. Hillier, F.S. "X-and R-chart control limits based on a small number of subgroups", Journal of Quality Technology, 1(1), pp. 177-26(1969).

22. Hillier, F.S. "Small sample probability limits for the range chart", Journal of the American Statistical Association, 62(320), pp. 1488-1493 (1967).

23. Wheeler, D.J. and David, S., Understanding Statistical Process Control, SPC PRESS (Statistical Process Control), 3rd Edition, ISBN-13, 978-0945320692 (1992).

24. Quevedo, V., Vegas, S., and Vining, G. "A tutorial on an iterative approach for generating Shewhart control limits", Quality Engineering, 28(3), pp. 305$312(2016)$.
25. Djauhari, M.A., Sagadavan, R., and Li, L.S. "Monitoring multivariate process variability when sub-group size is small", Quality Engineering, 28(4), pp. 429-440 (2016).

26. Abbasi, S.A., Ahmad, S., and Riaz, M. "On enhanced sensitivity of nonparametric EWMA control charts for process monitoring", Scientia Iranica, Transaction E, Industrial Engineering, 24(1), p. 424 (2017).

27. Mahmood, T., Nazir, H.Z., Abbas, N., Riaz, M., and Ali, A. "Performance evaluation of joint monitoring control charts", Scientia Iranica, 24(4), pp. 2152-2163 (2017).

28. Ajadi, J.O. and Riaz, M. "New memory-type control charts for monitoring process mean and dispersion", Scientia Iranica, Transactions E., Industrial Engineering, 24(6), pp. 3423-3438 (2017).

29. Abujiya, M.R., Lee, M.H., and Riaz, M. "New EWMA $\mathrm{S}$ sup 2 ^ control charts for monitoring of process dispersion", Scientia Iranica, Transactions E., Industrial Engineering, 24(1), p. 378 (2017).

30. Mukherjee, A. "Distribution-free phase-II exponentially weighted moving average schemes for joint monitoring of location and scale based on subgroup samples", The International Journal of Advanced Manufacturing Technology, 92(1-4), pp. 101-116 (2017).

31. Ansorena, Í. L. "Statistical process control and quality of service at seaports", International Journal of Productivity and Quality Management, 24(2), pp. 165-176 (2018).

32. Hussain, S., Song, L., Ahmad, S., and Riaz, M. "New interquartile range EWMA control charts with applications in continuous stirred tank rector process", Arabian Journal for Science and Engineering, 44(3), pp. 2467-2485 (2019).

33. Abtew, M.A., Kropi, S., Hong, Y., and Pu, L. "Implementation of statistical process control (SPC) in the sewing section of garment industry for quality improvement", Autex Research Journal, 18(2), pp. 160-172 (2018).

34. Huberts, L.C., Schoonhoven, M., and Does, R.J. "The effect of continuously updating control chart limits on control chart performance", Quality and Reliability Engineering International, 35(4), pp. 1117-1128 (2019).

35. Montgomery, D.C., Introduction to Statistical Quality Control, John Wiley and Sons, New York (2009).

36. Chakraborti, S. "Run length, average run length and false alarm rate of Shewhart X-bar chart: Exact derivations by conditioning", Communications in StatisticsSimulation and Computation, 29(1), pp. 61-81 (2000).

37. Sekhon, B.S. "Food nanotechnology-an overview", Nanotechnology, Science and Applications, 3, p. 1 (2010).

38. Ahmed, T., Imdad, S., Yaldram, K., and Raza, S.M. "Awareness and attitude about nanotechnology in Pakistan", Journal of Nano Education, 7(1), pp. 44-51 (2015). 
39. Razzaq, A., Ammara, R., Jhanzab, H.M., Mahmood, T., Hafeez, A., and Hussain, S. "A novel nanomaterial to enhance growth and yield of wheat", Journal of Nano Science and Technology, 2(1), pp. 55-58 (2016).

\section{Biographies}

Qurat-Ul-Ain Khaliq is currently a $\mathrm{PhD}$ student in Statistics from Department of Statistics, Allama Iqbal Open University, Islamabad. She holds the position of Lecturer (Statistics) at Higher Education Department, Punjab. Her current research interests include statistical process control, non-parametric techniques, linear profile, and use of sampling techniques in SPC.

Muhammad Riaz obtained his PhD degree in Statistics from Institute for Business and Industrial Statistics, University of Amsterdam, The Netherlands in
2008. He holds the position of Professor at the Department of Mathematics and Statistics, King Fahad University of Petroleum and Minerals, Dhahran, Saudi Arabia. His current research interests include statistical process control, non-parametric techniques, and experimental designs.

Irshad Ahmad Arshad is currently holding the position of Chairman, Department of Statistics. Allama Iqbal Open University, Islamabad, Pakistan. His research interests include statistical process control, non-parametric techniques, and econometrics.

Shahla Gul is currently a PhD student in Education from University of Arid Agriculture, Rawalpindi. Her current research interests include statistical process control, educational technology, and research methodology. 